\title{
Case Report: Anterior Cruciate Ligament Calcification in a Patient With Chondrocalcinosis: Micro-Computed Tomography Presentation
}

\author{
Alberto Grassi ${ }^{1 *}$, Giacomo Dal Fabbro ${ }^{1}$, Milena Fini ${ }^{2}$, Stefano Zaffagnini ${ }^{1}$ and \\ Annapaola Parrilli ${ }^{3 *}$
}

${ }^{1}$ IRCCS Istituto Ortopedico Rizzoli, 2nd Orthopaedic and Traumatologic Clinic, Bologna, Italy, ${ }^{2}$ IRCCS Istituto Ortopedico Rizzoli, RIT Department, Surgical Sciences and Technologies, Bologna, Italy, ${ }^{3}$ Empa - Swiss Federal Laboratories for Materials Science and Technology, Center for X-ray Analytics, Dübendorf, Switzerland

\section{OPEN ACCESS}

Edited by:

Claudia Di Bella

The University of Melbourne, Australia

Reviewed by:

Piotr Zarychta,

Silesian University of

Technology, Poland

Nikolaos Paschos,

Massachusetts General Hospital and Harvard Medical School,

United States

*Correspondence: Alberto Grassi

alberto.grassi@ior.it

Annapaola Parrilli

annapaola.parrilli@empa.ch

Specialty section:

This article was submitted to

Orthopedic Surgery,

a section of the journal

Frontiers in Surgery

Received: 13 March 2021

Accepted: 29 June 2021

Published: 29 July 2021

Citation:

Grassi A, Dal Fabbro G, Fini M, Zaffagnini S and Parrilli A (2021) Case

Report: Anterior Cruciate Ligament

Calcification in a Patient With

Chondrocalcinosis: Micro-Computed

Tomography Presentation.

Front. Surg. 8:680234.

doi: 10.3389/fsurg.2021.680234
In this case report, an incidental postoperative diagnosis of anterior cruciate ligament $(A C L)$ calcification, associated with calcification of posterior cruciate ligament (PCL) and lateral meniscus insertions, was made using micro-computed tomography ( $\mu \mathrm{CT}$ ) technology in a knee specimen obtained during a total knee replacement (TKR) surgery due to painful tri-compartmental osteoarthritis $(\mathrm{OA})$ with chondrocalcinosis signs at preoperative $\mathrm{X}$-ray. Anterior cruciate ligament calcification is an uncommon finding, and conventional X-ray and MRI are not so helpful in its identification. $\mu \mathrm{CT}$ scan, in contrast, is of interest because it provides highly spatial three-dimensional information with excellent visualization of bones and calcifications. The $\mu \mathrm{CT}$ technology used in this case report allowed us to perform a detailed analysis and a 3-D reconstruction of the calcium pyrophosphate dihydrate (CPPD) crystal deposition about the knee without the need to section the specimens into slice as performed in previous studies. The 3-D model obtained with $\mu \mathrm{CT}$ scan permits to gain more insight into the shape of the calcification within the fibers of the ligamentous structures of the joint.

Keywords: knee, anterior cruciate ligament, chondrocalcinosis, calcification, Micro-CT (computed tomography), $\mu \mathrm{CT}$ and $3 \mathrm{D}$ reconstruction

\section{INTRODUCTION}

Calcification of the anterior cruciate ligament (ACL), described first in a cadaveric case by Steinbach et al. (1), is a rare clinical entity, and only few cases have been provided in the literature $(2,3)$. Although some occurrences were reported, it is still not clear if ACL calcification is related to any previous injury or surgery (4-7). Several risk factors for ACL calcification have been analyzed: Thyroid disorders, diabetes, and PTH disorders have been proposed, without being confirmed so far (8).

Chondrocalcinosis is a common disorder in the elderly $(9,10)$ characterized by calcium pyrophosphate dihydrate (CPPD) crystal deposition in and around articular tissues (11-13) and represents a risk factor for the development of calcification of ACL $(3,14-17)$. Conventional $\mathrm{X}$-ray is the most commonly used imaging method for the visualization of intra-articular calcium 
crystal deposition and allows us to recognize CPPD deposition in fibrocartilage, but it lacks in sensitivity limiting an insight into burden and localization (18). MR imaging is limited in the distinction between calcification of cartilage and tissues from other abnormalities (19). Computed tomography (CT) scan, rather, is of interest because it provides highly spatial threedimensional (3D) information with excellent visualization of bones and calcifications $(11,17,20)$. Due to the resolution of these clinical systems and to the challenge in differentiating CPPD deposition from other lesions $(18,19)$, 3D reconstruction of the knee joint by both CT and MR is usually limited to the reconstruction of the bone segments and used as tools for improving the planning or the outcomes of the knee surgery (21).

The clinical implications of calcification of ACL and its association with ligament degeneration and development of knee osteoarthritis (OA) are still not fully acknowledged because of the lack of clear studies $(3,15)$.

The aim of the present case report was to describe the tridimensional distribution pattern of ACL calcification in the context of chondrocalcinosis, using a micro-computed tomography $(\mu \mathrm{CT})$ technology. The hypothesis was that using this method, it would be possible to investigate the shape and course of calcifications within the ligament fibers and from multiple spatial view orientations.

\section{CASE DESCRIPTION}

A human specimen of tibial plateau and femoral intercondylar notch including ACL, posterior cruciate ligament (PCL), and meniscal insertions (lateral meniscus) was obtained from a patient with diagnosis of chondrocalcinosis during the surgery for total knee replacement (TKR) due to painful tricompartmental knee OA. The specimen was scanned with a $\mu \mathrm{CT}$ system, and a $3 \mathrm{D}$ reconstruction of the specimen was built. Informed consent was obtained from the patient included in the case report.

\section{Patient Characteristics}

A 72-year-old housewife presented with a chronic and growing right knee pain for more than 10 years with important functional limitation during normal daily activities. Clinical examination showed a swollen knee with varus malalignment. An acute pain was evoked by palpation of the joint space narrowing. The passive range of motion was restricted from $0^{\circ}$ to $90^{\circ}$. The knee were stable under antero-posterior and varus-valgus stress. The X-ray examination showed a tri-compartmental OA of the knee, especially the medial tibia-femoral compartment and patella-femoral compartment. Moreover, X-ray examination showed chondrocalcinosis of the medial and lateral meniscus (Figure 1). Patient denied prior knee trauma or surgery. In her history, she reported a post-thyroidectomy hypothyroidism and osteoporosis. Previous elbow and shoulder radiographs obtained for minor traumatic events revealed the presence of $\mathrm{CPPD}$ at the level of rotator cuff and writs flexors insertion at medial epicondyle as well (Figure 2). She was in therapy with cardioaspirin, pantoprazole, ibandronic acid, vitamin D, and levothyroxine. Blood test revealed a normal blood count and

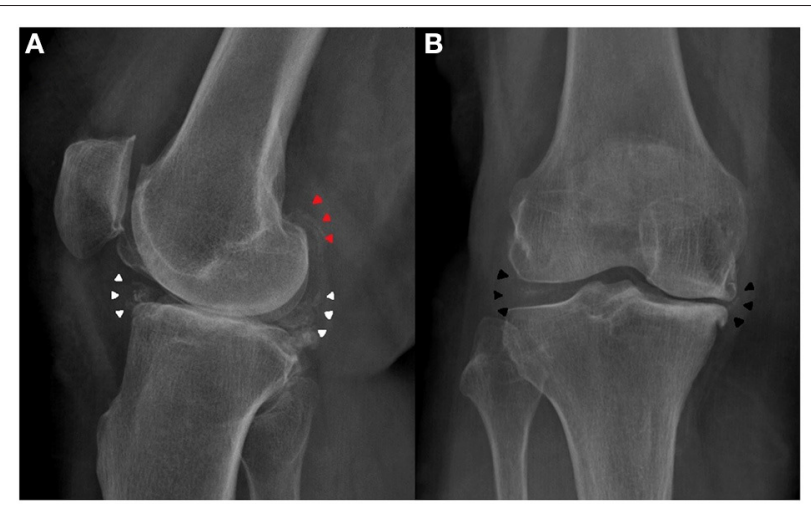

FIGURE 1 | Preoperative knee X-ray of the patient. (A) Lateral view showed meniscus (white arrowheads) and gastrocnemius tendon (red arrowheads) calcification. (B) Antero-posterior view showed medial and lateral meniscus calcification (black arrowheads).

normal blood levels of electrolytes, calcium, and phosphorus. Thus, the present patient was indicated for TKR surgery. Following the surgery, a partial weight bearing was set for 2 weeks. Physical therapy and range of motion exercise started 3 days after the surgery. At 3 weeks after the surgery, the patient was cleared to full weight-bearing. At her final follow-up, 1 year postoperatively, the patient was satisfied with the outcomes and still doing well with no complaints of pain, presenting an active range of motion from $0^{\circ}$ to $115^{\circ}$.

\section{Specimen Preparation}

During the planned surgery of a mechanically aligned TKR, a standard medial parapatellar approach was performed, and the specimen was obtained by cutting the intercondylar notch in a box-shaped fashion using an osteotome, in order to preserve the femoral ACL insertion. The tibial plateau was cut with an oscillating saw as planned during the TKR. Menisci were removed from the tibial plateau leaving only the most peripheral tibial attachments, as the standard technique for TKR in order to have the optimal visualization of tibial plateau during the cut. TKR was performed in a standard fashion, and specimen harvesting had no consequence on the TKR technical execution or other intraoperative choices such as implant choice, degree of constrain, soft tissue balancing, blood loss, and surgical time.

The harvested specimen was kept at full knee extension position and fixed in 10\% neutral-buffered formalin for $48 \mathrm{~h}$ to stabilize the tissue components. After washing in distilled water for $24 \mathrm{~h}$, the sample was fully dehydrated using a graded series of ethanol $(70,80,90$, and $100 \%)$, by immersing in hexamethyldisilazane and air.

\section{Micro-Computed Tomography Analysis}

The dry sample was scanned with the $\mu \mathrm{CT}$ system Skyscan 1176 (Bruker microCT, Kontich, Belgium) at a source voltage of $45 \mathrm{kV}$, a source current of $550 \mu \mathrm{A}$, with an aluminum filter of $0.2 \mathrm{~mm}$, and an exposure of $185 \mathrm{~ms}$. The nominal resolution was set at $17.5 \mu \mathrm{m}$. For the reconstruction in tomographic 


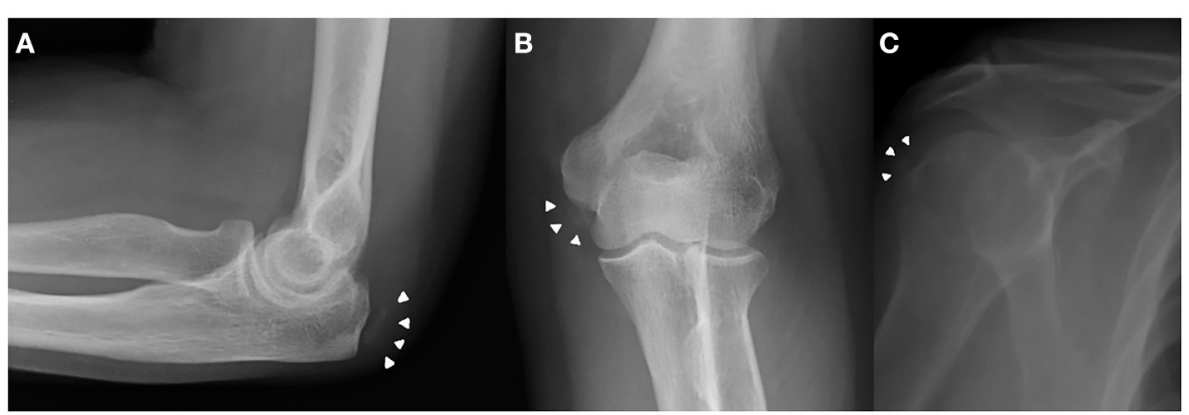

FIGURE 2 | Previous elbow and shoulder X-ray obtained for minor traumatic events revealed the presence of CPPD crystal deposition (white arrowheads) at the level of writs flexors insertion at medial epicondyle $(\mathbf{A}, \mathbf{B})$ and rotator cuff $(\mathbf{C})$ as well.

2D sections, we used the dedicated NRecon software (version 1.6.10.4, Bruker micro-CT) applying corrections for specific misalignment, a low ring artifact reduction, and beam hardening. 3D model of the knee and its components (bone, cartilage, ACL, PCL, and menisci) was created using Avizo software (ThermoFisher Scientific). In order to distinguish the different knee joint components, we both divided the knee in volumes of interest and used global threshold depending on the different X-ray absorption of mineralized and soft tissues. The components were then virtually colored in the overall $3 \mathrm{D}$ model to enhance their differentiation.

\section{LIGAMENTS AND MENISCI APPEARANCE}

The post-acquisition elaboration of the specimen allowed to build a 3D model of the tibial plateau, ACL, PCL tibial insertion, and lateral meniscus posterior and anterior horn insertions (Figure 3 and Supplementary Video 1). According to the views from coronal, axial, and sagittal planes, multiples intra-substance calcifications within the whole ACL structure and diameter were appreciated. Their shape was linear and sinuous, running parallel to ACL fibers. With the $3 \mathrm{D}$ reconstruction and by varying the view of inclination angle, it was possible to follow the multiple calcifications from the tibial insertion to the femoral origin, thus suggesting the shape and course of the ligament itself.

Examining the specimen on the sagittal plane, it was appreciated the linear course of the calcifications along the ACL (Figure 4A). A similar pattern was present in the distal part of the PCL included in the specimen (Figure 4B). Differently, on the coronal plane centered on the tibial insertion (Figure 4C), it was possible to note the calcifications with an oval shape, due to the virtual cut along their shorted diameter.

A similar presentation was noted for the flat menisco-tibial ligament at the posterior horn insertions of the lateral meniscus included in the specimen where calcifications were linear on sagittal cuts (Figure 4D). Differently, calcifications within the triangular meniscal remnant were observed to be round or oval in shape in all planes (Figures 4E,F).

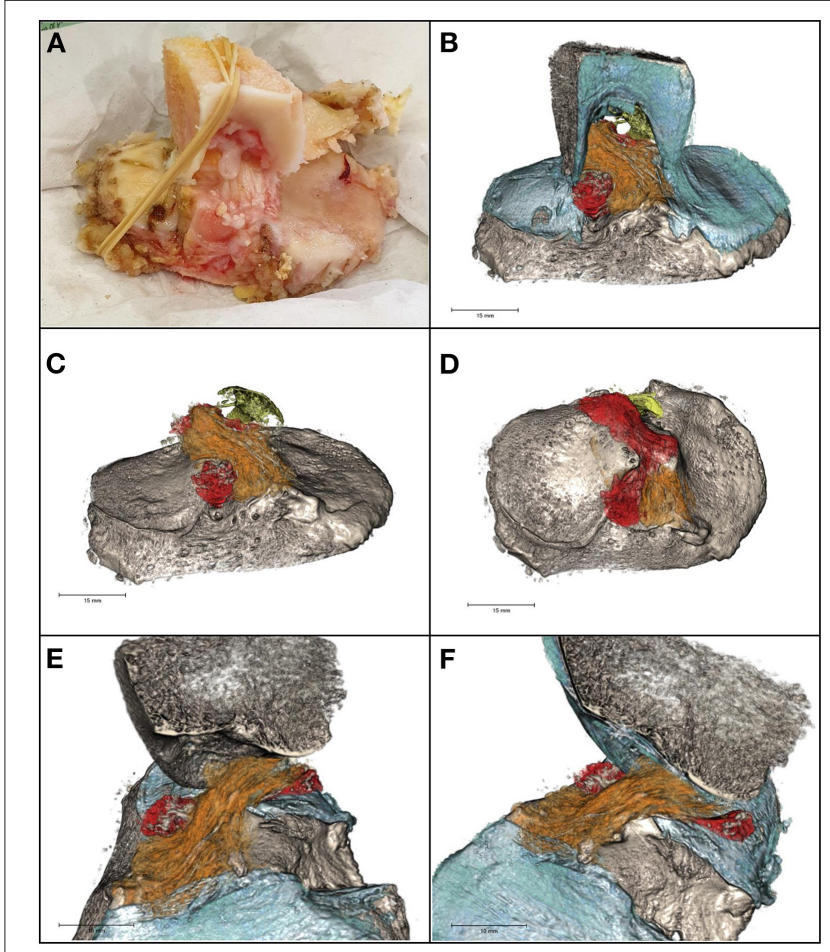

FIGURE 3 | (A) The specimen. (B) 3D model of intercondylar notch, tibial plateau, cartilage (blue), ACL (orange) and PCL (yellow) tibial insertions, and lateral meniscus posterior and anterior horn insertions (red). Calcifications (gray) within the cruciate ligaments and the meniscal insertions supplemental fibers were linear in shape. (C) Antero-posterior view of ACL, PCL, and lateral meniscus tibial insertions. (D) Axial view of ACL, PCL, and lateral meniscus tibial insertions. (E,F) Sagittal view of the tibial plateau, ACL, and lateral meniscus tibial insertions.

\section{DISCUSSION}

In this case report, we described a patient with primary knee OA and chondrocalcinosis who underwent total knee arthroplasty in whom an incidental postoperative diagnosis of ACL calcification was made using $\mu \mathrm{CT}$ scan. 

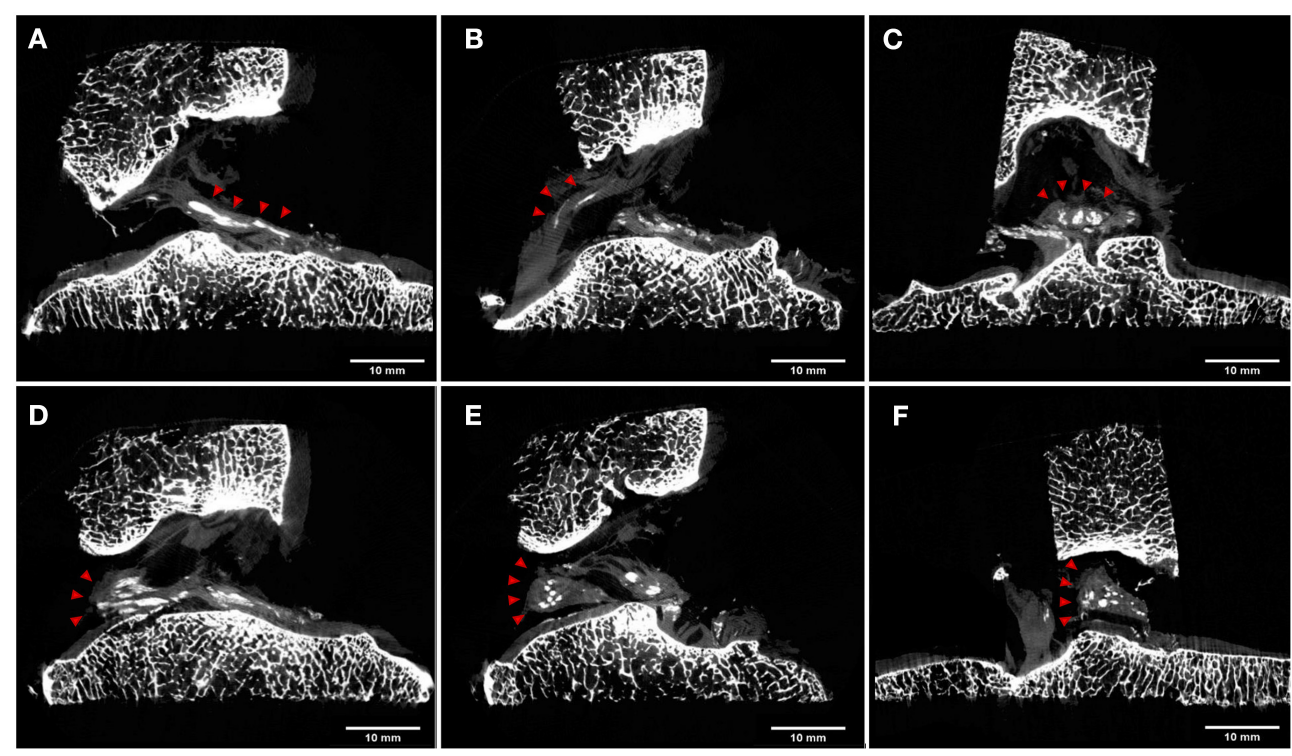

FIGURE 4 | $\mu$ CT cross sections. Calcifications are highlighted with red arrowheads. Sagittal views of ACL (A) and PCL (B) showed a linear pattern of the cruciate ligament calcifications. On the coronal plane, calcifications of ACL were characterized by an oval shape due to the virtual cut along their shortened diameter (C). Sagittal views of lateral meniscus posterior horn insertion and flat menisco-tibial ligament (D) showed linear pattern calcifications, while the triangular meniscal remnant showed round or oval calcification both in the sagittal (E) and in the coronal (F) view.

The main finding of this case report was that $\mu \mathrm{CT}$ technology permits to obtain a 3D analysis of intra-articular calcium crystal deposition within ligament fibers, without the need to section the specimen into slice as performed in previous cadaveric studies to analyze the involvement of ACL in CPPD crystal deposition $(15,16)$. Abreu et al. and Dirim et al. analyzed ACL calcification with high-spatial-resolution radiography (Faxitron; HP; McMinnville, OR, United States) of the slices obtained from sectioning frozen knee specimens and confirmed CPPD crystal deposition using hematoxylin-eosin on histologic analysis; the authors reported the calcification of ACL, respectively, in two $(50 \%)$ of four and in five $(62.5 \%)$ of eight knee specimens with CPPD deposition $(15,16)$. On the other hand, standard $\mathrm{X}$-ray and MRI seemed to reveal evidence of calcification in meniscal cartilage and hyaline cartilage, respectively, while they may not be helpful in the evaluation of calcification within ACL $(11,20)$. In a case report of 2016, Hayashi et al. described an incidental diagnosis of ACL calcification in a patient with chondrocalcinosis: While the CPPD crystal deposition within meniscal fibro cartilage was recognized on $\mathrm{X}$-ray, the ACL calcification was not visualized on X-ray but only at CT of the knee (3). Even in the presented case, the calcification of ACL was visible not on X-ray but only on $\mu \mathrm{CT}$ examination. In a CT imaging in vivo study of 2015, Misra et al. reported the calcification of ACL in 10 of 24 knees with radiographic chondrocalcinosis, providing the potential utility of CT scan in the evaluation of intra-articular calcium crystal deposition (17).

The 3D analysis provided by $\mu \mathrm{CT}$ scan technology in the current case report showed a linear and sinuous pattern of the ACL calcification, with the shape running parallel to the ligamentous fibers. A similar pattern was reported in the studies of Abreu et al. (16) and Dirim et al. (15), in which the authors described the calcifications linear in shape and elongated in the plane of the fibers of the ligament (Figure 4).

Moreover, in the current case report, a linear pattern of calcification within the PCL fibers was observed as well on microCT imaging (Figure 4B); in the cadaveric studies of Dirim et al. (15), all five knees with ACL calcification presented an associated PCL calcification, characterized by a linear shape very similar to that described in the current case report.

The calcifications in the lateral meniscal remnant in the current case report were seen to be punctate and oval (Figures 4E,F). A similar pattern was reported by previous cadaveric studies, in which meniscal calcifications were described as round or oval in shape and aggregated in clusters (15, 16). Differently, a calcification pattern similar to ligamentous structures such as ACL and PCL was observed in the meniscotibial ligament at the level of lateral meniscus posterior horn, however with a different fiber orientation compared to cruciate ligament calcifications (Figure 4D). In authors' opinion, this finding could be explained because these calcifications developed within the supplemental fibers of the meniscal root attachment, which have an elongated linear shape (22).

In conclusion, the implication of the presented case and the few studies that the authors founded in the literature suggested that the involvement of ACL in the CPPD deposition disease is frequent, but the conventional X-ray and MRI could not be helpful in its identification. $\mu \mathrm{CT}$ scan enables the detection of calcium crystal in deeper intra-articular structure like ACL and represents an important tool to better understand the morphology of calcifications without 
the potential artifacts of classic histology. Moreover, $\mu \mathrm{CT}$ scan allows us to investigate in deep the shape and course of calcifications within the ligament fibers from multiple spatial view orientations and in a $3 \mathrm{D}$ manner. Further studies describing more cases from other patients are needed to confirm the potential of the $\mu \mathrm{CT}$ scan in the analysis of the knee intra-articular structure.

\section{DATA AVAILABILITY STATEMENT}

The raw data supporting the conclusions of this article will be made available by the authors, without undue reservation.

\section{ETHICS STATEMENT}

Ethical review and approval was not required for the study on human participants in accordance with the local legislation and institutional requirements. The patients/participants provided their written informed consent to participate in this study.

\section{REFERENCES}

1. Steinbach LS, Resnick D. Calcium pyrophosphate dihydrate crystal deposition disease revisited. Radiology. (1996) 200:19. doi: 10.1148/radiology.200.1.8657894

2. Tsujii A, Tanaka Y, Yonetani Y, Iuchi R, Shiozaki Y, Horibe S. Symptomatic calcification of the anterior cruciate ligament: a case report. Knee. (2012) 19:223-5. doi: 10.1016/j.knee.2011.05.008

3. Hayashi $\mathrm{H}$, Fischer $\mathrm{H}$. Incidental anterior cruciate ligament calcification: case report. J Radiol Case Rep. (2016) 10:20-7. doi: 10.3941/jrcr.v10i3. 2319

4. Taggart TF, Kumar A, Suvarna SK, Bickerstaff DR. Osseous metaplasia as a cause of loss of extension after anterior cruciate ligament reconstruction. Arthroscopy. (2001) 17:405-7. doi: 10.1053/jars.2001. 21830

5. Batra GS, Harrison JW, Clough TM, Paul AS. Failure of anterior cruciate ligament reconstruction following calcification of the graft. Knee. (2002) 9:245-7. doi: 10.1016/s0968-0160(02)00021-2

6. Seeger LL, Sako E, Motamedi K, Levine BD, Hame SL. Intraarticular calcifications following arthroscopic ACL reconstruction: prevalence and possible significance. Skeletal Radiol. (2014) 43:209-12. doi: 10.1007/s00256-013-1773-6

7. Minezaki T, Tomatsu T, Hanada K. Calcium pyrophosphate dihydrate crystal deposition disease after anterior cruciate ligament reconstruction. Arthroscopy. (1998) 14:634-6. doi: 10.1016/s0749-8063(98) 70064-7

8. Harvie P, Pollard TC, Carr AJ. Calcific tendinitis: natural history and association with endocrine disorders. J Shoulder Elbow Surg. (2007) 16:16973. doi: 10.1016/j.jse.2006.06.007

9. Ramonda R, Musacchio E, Perissinotto E, Sartori L, Punzi L, Corti MC, et al. Prevalence of chondrocalcinosis in Italian subjects from northeastern Italy. The ProVA (PROgetto Veneto Anziani) study. Clin Exp Rheumatol. (2009) 27:981-4.

10. Mccarty DJ Jr, Hogan JM, Gatter RA, Grossman M. Studies on pathological calcifications in human cartilage. I. Prevalence and types of crystal deposits in the menisci of two hundred fifteen cadavera. J Bone Joint Surg. (1966) 48:30925 .

11. Touraine S, Ea HK, Bousson V, Cohen-Solal M, Laouisset L, Chappard C, et al. Chondrocalcinosis of femoro-tibial and proximal tibio-fibular joints in
Written informed consent was obtained from the individual(s) for the publication of any potentially identifiable images or data included in this article.

\section{AUTHOR CONTRIBUTIONS}

AG, GD, and AP wrote the first draft of the article. AG and SZ performed the surgery. AG, SZ, and AP interpreted the results. AP designed and performed the $\mu \mathrm{CT}$ analysis. AG, GD, SZ, MF, and AP critically reviewed the drafts. All authors contributed to the article and approved the submitted version.

\section{SUPPLEMENTARY MATERIAL}

The Supplementary Material for this article can be found online at: https://www.frontiersin.org/articles/10.3389/fsurg. 2021.680234/full\#supplementary-material

Supplementary Video $1 \mid \mu C T$ 3D model of the tibial plateau, ACL, PCL tibial insertion, and lateral meniscus posterior and anterior horn insertions.

cadaveric specimens: a high-resolution CT imaging study of the calcification distribution. PLoS ONE. (2013) 8:e54955. doi: 10.1371/journal.pone. 0054955

12. Zhang W, Doherty M, Bardin T, Barskova V, Guerne PA, Jansen $\mathrm{TL}$, et al. European League Against Rheumatism recommendations for calcium pyrophosphate deposition. Part I: terminology and diagnosis. Ann Rheum. Dis. (2011) 70:563-70. doi: 10.1136/ard.2010. 139105

13. Yang BY, Sartoris DJ, Resnick D, Clopton P. Calcium pyrophosphate dihydrate crystal deposition disease: frequency of tendon calcification about the knee. $J$ Rheumatol. (1996) 23:883-8.

14. Ryu $\mathrm{K}$, Iriuchishima $\mathrm{T}$, Oshida $\mathrm{M}$, Kato $\mathrm{Y}$, Saito $\mathrm{A}$, Imada $\mathrm{M}$, et al. The prevalence of and factors related to calcium pyrophosphate dihydrate crystal deposition in the knee joint. Osteoarthr Cartil. (2014) 22:9759. doi: 10.1016/j.joca.2014.04.022

15. Dirim B, Resnick D, Abreu M, Wangwinyuvirat M, Trudell DJ, Haghighi P. Relationship between the degeneration of the cruciate ligaments and calcium pyrophosphate dihydrate crystal deposition: anatomic, radiologic study with histologic correlation. Clin Imaging. (2013) 37:342-7. doi: 10.1016/j.clinimag.2012. 03.002

16. Abreu M, Johnson K, Chung CB, De Lima JE Jr, Trudell D, Terkeltaub R, et al. Calcification in calcium pyrophosphate dihydrate (CPPD) crystalline deposits in the knee: anatomic, radiographic, MR imaging, and histologic study in cadavers. Skelet Radiol. (2004) 33:392-8. doi: 10.1007/s00256-0040767-9

17. Misra D, Guermazi A, Sieren JP, Lynch J, Torner J, Neogi T, et al. CT imaging for evaluation of calcium crystal deposition in the knee: initial experience from the Multicenter Osteoarthritis (MOST) study. Osteoarthr Cartil. (2015) 23:244-8. doi: 10.1016/j.joca.2014.10.009

18. Wenham CY, Grainger AJ, Conaghan PG. The role of imaging modalities in the diagnosis, differential diagnosis and clinical assessment of peripheral joint osteoarthritis. Osteoarthr Cartil. (2014) 22:1692-702. doi: 10.1016/j.joca.2014. 06.005

19. Gersing AS, Schwaiger BJ, Heilmeier U, Joseph GB, Facchetti L, Kretzschmar M, et al. Evaluation of chondrocalcinosis and associated knee joint degeneration using MR imaging: data from the osteoarthritis initiative. Eur Radiol. (2017) 27:2497-506. doi: 10.1007/s00330-0164608-8 
20. Miksanek J, Rosenthal AK. Imaging of calcium pyrophosphate deposition disease. Curr Rheumatol Rep. (2015) 17:20. doi: 10.1007/s11926-015-0496-1

21. Zarychta P. A new approach to knee joint arthroplasty. Comput Med Imaging Graph. (2018) 65:32-45. doi: 10.1016/j.compmedimag.2017. 07.002

22. LaPrade CM, Ellman MB, Rasmussen MT, James EW, Wijdicks CA, Engebretsen L, et al. Anatomy of the anterior root attachments of the medial and lateral menisci: a quantitative analysis. Am J Sports Med. (2014) 42:2386-92. doi: 10.1177/036354651 4544678

Conflict of Interest: The authors declare that the research was conducted in the absence of any commercial or financial relationships that could be construed as a potential conflict of interest.
Publisher's Note: All claims expressed in this article are solely those of the authors and do not necessarily represent those of their affiliated organizations, or those of the publisher, the editors and the reviewers. Any product that may be evaluated in this article, or claim that may be made by its manufacturer, is not guaranteed or endorsed by the publisher.

Copyright (c) 2021 Grassi, Dal Fabbro, Fini, Zaffagnini and Parrilli. This is an open-access article distributed under the terms of the Creative Commons Attribution License (CC BY). The use, distribution or reproduction in other forums is permitted, provided the original author(s) and the copyright owner(s) are credited and that the original publication in this journal is cited, in accordance with accepted academic practice. No use, distribution or reproduction is permitted which does not comply with these terms. 